\title{
Sous la bannière de la science du folklore
}

Territoire, histoire et identité au XIX ${ }^{\mathrm{e}}$ siècle

\section{Mercedes Gómez-García Plata}

\section{(2) OpenEdition}

12 Journals

\section{Édition électronique}

URL : http://journals.openedition.org/agedor/4867

DOI : 10.4000 /agedor.4867

ISSN : 2104-3353

Éditeur

Laboratoire LISAA

Référence électronique

Mercedes Gómez-García Plata, «Sous la bannière de la science du folklore », L'Âge d’or [En ligne], 12 I

2019, mis en ligne le 01 octobre 2020, consulté le 15 octobre 2020. URL : http://

journals.openedition.org/agedor/4867 ; DOI : https://doi.org/10.4000/agedor.4867

Ce document a été généré automatiquement le 15 octobre 2020.

L'Âge d'or. Images dans le monde ibérique et ibéricoaméricain 


\section{Sous la bannière de la science du folklore}

Territoire, histoire et identité au XIX ${ }^{\mathrm{e}}$ siècle

Mercedes Gómez-García Plata

1 L'intérêt érudit pour la collecte et l'étude de la culture populaire est un mouvement intellectuel qui se développe en Europe du Nord à la charnière des XVIII ${ }^{\mathrm{e}}$ et XIX ${ }^{\mathrm{e}}$ siècles. J. G. Herder et ses successeurs, fondateurs de la Volkskunde - les frères Grimm, L. Jahn, entre autres -, sont à l'origine d'un formidable élan de collecte de traditions populaires langagières, littéraires et coutumières dont le but est d'inventorier l'héritage du passé afin de promouvoir le patriotisme culturel. Dans cette perspective, la culture populaire, considérée comme "archives» des nations en construction, est mise au service de l'édification du sentiment national pour en être son fondement culturel, l'aspect idéologique important plus que le critère scientifique.

2 Dans la deuxième moitié du XIX ${ }^{e}$ siècle, l'étude de la culture populaire, désormais désignée sous le nom de folklore - depuis l'introduction du néologisme par W. J. Thoms en 1845 - profite de la révolution darwinienne et de la formation du discours anthropologique qui change la nature de son contrat épistémologique. Doté d'un cadre institutionnel, la Folk-Lore Society, le folklore, en voie d'autonomisation disciplinaire, a pour finalité de participer non plus à la documentation et à la consolidation du sentiment national, mais à la connaissance de l'évolution de la culture humaine dans une perspective universelle. À l'instar de la Volkskunde dont l'enjeu philologicohistorique ${ }^{1}$ avait dominé la première moitié du XIX ${ }^{e}$ siècle, les postulats théoriques et institutionnels de la Folk-Lore Society marquent le mouvement folkloriste européen qui se développe à partir des années 1870 .

3 L'Espagne, qui avait été sous-représentée durant la première étape de valorisation et de collecte de la culture populaire du début de siècle, participe pleinement à cet élan folkloriste européen grâce aux efforts et au volontarisme d'un homme: Antonio Machado y Álvarez. Cet intellectuel, fils du naturaliste Antonio Machado y Núñez et petit-neveu de Agustín Durán, le compilateur du Romancero general, entend importer et institutionnaliser en Espagne le folklore scientifique tel qu'il est défini par l'école 
anthropologique britannique. Cependant, son héritage intellectuel et familial, ses convictions républicaines fédéralistes et son inquiétude pour régénérer la vie intellectuelle espagnole le conduisent à adapter ce modèle exogène à la réalité sociale espagnole et à concevoir le folklore comme un outil scientifique capable d'influer sur la construction de l'imaginaire national qu'il souhaite laïc, démocratique et progressiste.

4 Après avoir présenté les caractéristiques de l'entreprise machadienne d'institutionnalisation du folklore dans ses dimensions scientifique et patriotique, cet article s'intéressera aux obstacles auxquels se heurte cette proposition de (re)construction de l'imaginaire national sur des bases démocratiques et fédératrices dans le contexte de la première décennie de la Restauration canoviste.

5 En apprenant, en 1880, par la Revue Celtique que la Folk-Lore Society a été créée à Londres afin de recueillir et d'étudier la culture populaire, A. Machado y Álvarez écrit à son secrétaire, G. L. Gomme, pour en devenir membre et recevoir les travaux édités jusqu'alors. Son objectif est de s'inspirer du modèle anglais pour fonder sa propre société de folklore tout en l'adaptant à la réalité territoriale et culturelle de l'Espagne. En effet, dans sa conception, le folklore qu'il considère comme un " proceso científico moderno regenerador ${ }^{2}$ doit devenir un outil au service de la reconstruction scientifique de l'histoire et de la culture espagnoles ${ }^{3}$. Afin de poursuivre son plan d'acclimatation du cadre théorique et institutionnel britannique, A. Machado y Álvarez rédige et publie à Séville, en novembre 1881, un petit opuscule intitulé, Bases del FolkLore Español, acte de fondation de sa société de folklore qu'il nomme El Folk-Lore Español. Cette charte constitue le principal instrument pour parachever l'hispanisation $\mathrm{du}$ modèle exogène. Si la définition de l'objet, des champs disciplinaires, de la méthodologie et l'obligation de publication des résultats sont similaires aux propositions britanniques, l'organisation territoriale de la société, sous forme de fédération des différents centres folkloriques qui seront établis dans les provinces historiques de l'Espagne, ainsi que la finalité patriotique de son contrat épistémologique consacrent la singularité de El Folk-Lore espagnol ${ }^{4}$.

6 Entre 1881 et 1886, A. Machado y Álvarez mène une intense activité théorique, méthodologique, éditoriale et prosélyte afin de promouvoir l'expansion de son réseau de sociétés folkloriques sur tout le territoire, condition essentielle pour mener à terme l'institutionnalisation du folklore comme discipline, volet scientifique de son projet de (re)construction de l'histoire et de la culture espagnoles. À partir de la publication des Bases del Folk-Lore Español, pour mettre en branle la constitution des différentes sociétés régionales, A. Machado y Álvarez s'appuie sur un réseau d'amitiés et de connaissances très disparate d'un point de vue idéologique, mais néanmoins très homogène quant à leur appartenance à l'élite bourgeoise intellectuelle : écrivains, érudits, professeurs d'université ou de La Institución Libre de Enseñanza. C'est ainsi que le 28 novembre 1881, El Folk-Lore Andaluz, première société régionale du réseau, est fondée par A. Machado y Álvarez, qui en devient son secrétaire, alors que la présidence, fonction plus prestigieuse, échoit à l'historien, également spécialiste de l'œuvre de Cervantès et membre du Parti Conservateur, José María Asencio y Toledo. Parmi les membres du conseil, on retrouve d'éminentes personnalités intellectuelles de la bourgeoisie sévillane de différentes tendances idéologiques (conservateurs, évolutionnistes, républicaines krausistes), alors que les membres honoraires sont les plus éminents folkloristes européens. 
7 El Folk-Lore Frexnense, centre régional folklorique d'Estrémadure, se constitue le 11 juin 1882, à Fregenal de la Sierra (Badajoz), à l'initiative de Luis Romero y Espinosa, fondateur du journal local, El eco de Fregenal, mais aussi ami et condisciple de A. Machado y Álvarez. La procédure de constitution de la société est la même que celle du centre andalou dont elle reprend le règlement. Ses membres honoraires sont des professeurs de la I.L.E. (Juan Uña et Joaquín Sama). El Folk-Lore Castellano, comprenant les deux Castilles, troisième société régionale de la fédération, voit le jour le 28 Novembre 1883, sous la présidence du poète Gaspar Núñez de Arce. Eugenio Olavarría y Huarte, proche intellectuellement de la I.L.E., est nommé secrétaire. Il est d'ailleurs, avec Gumersindo de Azcárate, autre professeur de la I.L.E., qui a aussi rejoint le centre régional, l'homme de confiance de A. Machado y Álvarez. La création du centre castillan semble donner une impulsion au mouvement fédératif, puisque quelques mois plus tard, le $1^{\text {er }}$ février 1884 , sous la présidence de la célèbre romancière, Emilia Pardo Bazán, le centre régional galicien, El Folk-Lore Gallego, est constitué à La Corogne ${ }^{5}$. En novembre 1884, c'est le centre basque, El Folk-Lore Vasco-Navarro, qui est fondé par Ramiro de Echave et Vicente de Arana, tous deux éditeurs de la revue Euskal-Erria ${ }^{6}$.

8 En dépit de l'intense campagne prosélyte menée par A. Machado y Álvarez auprès de personnalités locales afin de déployer le réseau de sociétés folkloriques sur tout le territoire péninsulaire (Aragon, Valence, Catalogne, Murcie, Asturies), insulaire (l'archipel des Canaries et des Baléares) et d'outre-mer (Puerto Rico, Cuba, archipel des Philippines) - conformément au deuxième article des statuts de l'entité fédérale, plus aucun autre centre régional n'est créé. En Catalogne, les élites catalanistes refusent d'adhérer au réseau machadien et préfèrent cultiver et revendiquer un entre-soi folklorique au sein de l'Association des excursionnistes de Catalogne (Associació d'excursions catalana), qui se dote, en mai 1885, d'une section dédiée au folklore ${ }^{7}$. Parallèlement à la mise en place du réseau des sociétés de El Folklore Español, A. Machado y Álvarez mène de front ses travaux de recherche et de collecte, ainsi qu'une intense activité éditoriale. Il fonde et dirige successivement deux revues folkloriques, $E l$ Folk-Lore Andaluz, en 1882, et El Folk-Lore Español. Biblioteca de la Tradiciones Populares Españolas, en 1883, destinées à accueillir les travaux des membres et des collaborateurs régionaux, nationaux et internationaux, soit à archiver, divulguer et échanger ${ }^{8}$.

Convaincu que la collecte méthodique est ce qui fait la différence entre un collectionneur de curiosités traditionnelles et un folkloriste, autrement dit entre le folklore comme passe-temps et le folklore comme discipline, A. Machado y Álvarez façonne ou adapte tout un arsenal d'outils pour collecter, inventorier, classer, décrire et archiver cet immense corpus, constituté de productions langagières, littéraires, de coutumes, croyances et rituels, etc., autrement dit tous les faits folkloriques énumérés dans le premier alinéa des statuts (Bases). Il publie ainsi, dans les revues folkloriques ou dans la presse nationale ou régionale, des questionnaires, des catalogues de classification, des protocoles d'expéditions folkloriques, d'usage de la photographie, de la sténographie ou de la notation musicale dans le but de permettre une représentation la plus fidèle possible des pratiques et des productions populaires qui ont vocation à constituer les archives scientifiques du folklore d'Espagne. Ce déploiement d'activités scientifiques et méthodologiques démontre qu'il ne fait aucun doute pour lui que le folklore, en tant qu'objet, constitue un ensemble de représentations et de rituels en relation avec un territoire et une histoire dont le rôle est de participer de la construction de l'imaginaire national. 
10 Sur le modèle du premier questionnaire du folkloriste français, Paul Sébillot, datant de 1880, A. Machado y Álvarez rédige son outil méthodologique emblématique: un questionnaire (interrogatorio) pour constituer une carte topographique tradtionnelle (mapa topográfico tradicional). Si le terrain de l'enquête est au départ limité à la province de Séville, le folkloriste espagnol compte bien l'étendre à toutes les contrées espagnoles, une fois l'établissement de toutes les sociétés locales et régionales achevé. $\mathrm{Au}$ moyen de ce questionnaire, il prétend interroger la géographie traditionnelle de façon à ce que chaque parcelle du territoire constitue une page animée de l'histoire nationale, autrement dit il entend utiliser la mémoire traditionnelle du territoire comme ressource historique. Ce projet de constitution de cartes topographiques traditionnelles présente, à son sens, deux bénéfices. En premier lieu, la connaissance du territoire national ou plutôt du territoire tel que le nomment, se l'approprient et le racontent ceux qui y vivent au quotidien est le facteur essentiel pour rechercher les sources d'une historiographie plus démocratique que l'histoire officielle, c'est-à-dire de (re)construire scientifiquement l'histoire espagnole, fondement $\mathrm{du}$ contrat épistémologique de sa société. En second lieu, la mise en relation étroite entre les hommes, le territoire et l'histoire permet aux individus d'être connaisseurs de leur passé, juges de leur présent et arbitres de leur destin. Cette caractéristique envisage le folklore, la connaissance du savoir populaire, dans une dimension dynamique et progressiste.

11 L'adaptation du cadre théorique et institutionnel britannique par A. Machado y Álvarez au contexte espagnol, tant du point de vue de l'organisation institutionnelle en société fédérale que de la finalité patriotique, fait du folklore machadien un projet collectif mobilisateur : la sauvegarde matérielle et la connaissance de la culture traditionnelle ont pour but de permettre à la nation espagnole, politiquement et idéologiquement divisée, d'accéder à la conscience de son unité dans sa diversité linguistique et régionale tout en contribuant à sa modernisation intellectuelle. Dans la conception machadienne, le folklore ne peut être une entreprise exclusive de l'élite intellectuelle et la participation de tous les Espagnols qui le peuvent et le souhaitent, sans distinction de classe, d'âge ou de sexe est requise. Il est convaincu que la connaissance de l'héritage traditionnel, façonné par la richesse de la diversité régionale et sociale permettra l'émergence du lien commun sur lequel se fonde l'unité nationale. Afin d'expliquer le folklore - objet et discipline - à la nation, il rédige et publie dans la presse régionale et nationale une série de textes scientifico-pédagogiques. Dans son exposé, A. Machado y Álvarez se livre, au fil des différents textes, à un véritable plaidoyer pour justifier, auprès de ses concitoyens, non seulement la réhabilitation de la culture populaire, par la collecte et l'étude, mais aussi sa revendication au service du projet patriotique. En s'appuyant sur des exemples concrets, il passe en revue histoire, langue et territoire, autrement dit les éléments constitutifs de la nation, qui déterminent son essence et son unité sur lesquelles se fonde le sentiment d'appartenance de ses membres, soit la nationalité ${ }^{9}$. Son discours a pour but de montrer que chaque région est différente, mais que chacune d'elles est une pièce essentielle à l'ensemble.

Dans le domaine linguistique, par exemple, il explique que le folklore aiderait à établir des dictionnaires qui décriraient la langue dans sa totalité. En effet, grâce à la connaissance des dialectes et des langues régionales, non seulement d'Espagne, mais aussi de toute la Péninsule, le locuteur pourrait emprunter des vocables catalans ou portugais plutôt que de faire des emprunts à la langue anglaise ou française, lorsqu'il 
n'y a pas de terme castillan approprié ${ }^{10}$. En ce qui concerne la connaissance du territoire ${ }^{11}$, A. Machado y Álvarez vante les mérites de l'élaboration de cartes topographiques traditionnelles, lesquelles présentent, selon lui, un double intérêt. En premier lieu, elles sont une façon d'articuler histoire et territoire, en scrutant les empreintes de l'histoire à travers le territoire, car l'étude des productions populaires attachées à un lieu permet de faire émerger la mémoire que le peuple, en tant que dépositaire et gardien des traditions nationales, a gardée du territoire comme principal théâtre des événements historiques. En second lieu, la maîtrise du territoire grâce aux connaissances traditionnelles peut avoir des applications très concrètes concernant la mise en valeur des ressources naturelles dans différents domaines comme l'orographie, les expéditions, les voyages, la connaissance de la faune et de flore, etc. ${ }^{12}$. En d'autres termes, par ces cartes topographiques traditionnelles, le peuple devient un sujet actif qui n'est pas seulement réductible à son rôle de conservatoire de la mémoire des traditions ${ }^{13}$.

13 En définitive, le folklore tel que A. Machado y Álvarez l'explique à la nation articule dans ses différentes représentations une interprétation populaire de l'histoire nationale sur une diversité territoriale et linguistique, éléments essentiels pour fonder et forger l'appartenance à la communauté nationale. En outre, étant donné qu'il demande aussi à ses compatriotes de participer à l'élaboration des cartes topographiques traditionnelles - par le biais de la collecte de matériaux sur le terrain, lesquels seront ensuite analysés et synthétisés par des spécialistes -, on peut dire qu'elles sont l'outil essentiel de son projet mobilisateur afin que tous les Espagnols soient des sujets actifs de la construction de l'imaginaire national.

On aura aussi remarqué, dans les explications qu'il livre à ses compatriotes, que le folkloriste ne présente pas le folklore comme une entreprise qui aurait pour but d'encourager la nostalgie d'une grandeur passée, car dans son système de pensée, la connaissance du passé est davantage un socle pour édifier l'unité nationale, une clé pour l'avenir ${ }^{14}$. En effet, le folklore machadien, qui réunit la tradition et le progrès, deux notions à priori antinomiques, mais mises au service l'une de l'autre, peut envisager, dans une synthèse neuve, à la fois le passé, le présent et le devenir de la nation. C'est aussi ce dépassement de l'antagonisme originel, cette conciliation de la dialectique entre la tradition et le progrès, qui est la garante de l'émergence de la conscience nationale. Cette conscience nationale ou de l'unité nationale, à son tour, révèle le bien commun, autrement dit le lien fraternel qui unit tous les enfants de la mère-patrie ${ }^{15}$.

En dépit d'une bonne médiatisation faite par les plus grands titres de la presse régionale et nationale ${ }^{16}$ aux discours, initiatives et projets proposés par A. Machado y Álvarez à ses concitoyens, on ne peut dire qu'il y ait eu un véritable élan national d'adhésion à son projet. La constitution des cartes topographiques traditionnelles reste à l'état d'ébauche, les différentes revues qu'il avait fondées cessent d'être publiées faute de financement et le réseau des sociétés régionales de folklore se désagrège progressivement entre 1886 et 1888 . Ces éléments signent l'échec de l'instutionnalisation du folklore dans ses dimensions scientifique et patriotique. Or, les outils forgés par A. Machado y Álvarez pour façonner le style de construction de l'imaginaire national, incarnés dans toutes ces initiatives, sont bien ceux que B. Anderson analyse dans son étude sur la formation des «imagined communities ${ }^{17}$. Ce 
paradoxe amène d'autant plus à s'interroger sur les causes de l'échec du projet machadien.

L'absence de soutien financier ou institutionnel de la puissance publique aux différentes demandes d'aides directes ou indirectes formulées par A. Machado y Álvarez (demande d'achat des volumes de la Biblioteca de la Tradiciones Populares Españolas pour les bibliothèques populaires afin de soutenir l'effort éditorial, demande de financement pour l'ouverture d'un musée folklorique à Madrid) a un rôle indiscutable dans l'échec machadien, en particulier en ce qui concerne ses causes matérielles, dont certaines sont aussi imputables au propre promoteur du projet. A. Machado y Álvarez n'a certes pas ménagé sa peine pour déployer une importante activité propagandiste, surtout de son discours, et prosélyte, pour établir son réseau de sociétés régionales, dans la presse espagnole, en particulier dans les meilleures revues intellectuelles de son temps ${ }^{18}$. Néanmoins, cet effort divulgateur aurait pu être amplifié. Dans ce domaine, sa volonté de préserver à tout prix la dimension scientifique du folklore, légitimation de son projet patriotique, l'a freiné dans une démarche vulgarisatrice qui aurait pu apporter une dimension plus concrète à ses projets et assurer ainsi une adhésion beaucoup plus large à ses propositions. L'inventivité dont il avait fait preuve dans le domaine méthodologique, en étant pionnier dans l'introduction des technologies modernes comme la photographie, lui a fait défaut à l'heure de diversifier les moyens et les supports de diffusion de son activité vulgarisatrice des représentations symboliques du folklore. Entre autres exemples, il aurait pu utiliser les photographies des scènes rituelles ou des jeux de l'enfance qu'il avait prises, éditées sous forme de carte postale et réservées à l'usage exclusif d'échange d'informations entre folkloristes pour réaliser des expositions ponctuelles dans le circuit des ateneos, sociétés culturelles ou bibliothèques populaires, ou pour en faire un plus grand tirage, lequel mis à la vente, aurait permis de lever des fonds pour financer d'autres projets ${ }^{19}$. De même, au plus mal de la période de financement de la Biblioteca de la Tradiciones Populares Españolas, il aurait pu réunir des miscellanées des matériaux collectés tels que chants, contes, adivinanzas ou pregones - dont certains avaient été publiés dans la presse -, lesquels édités sous formes de petits fascicules et vendus à des prix modiques auraient permis d'assurer une grande diffusion aux matériaux collectés et faire provision d'argent pour les revues spécialisées plus élitistes. En d'autres termes, il aurait dû moins compter sur la puissance du verbe pour convaincre ses concitoyens et plus se fier à celle des représentations visuelles afin d'apporter une dimension plus immédiate et concrète à ses projets, dont la plupart, à l'instar des cartes topographiques traditionnelles ne pouvaient produire des résultats qu'à long terme.

17 L'autre cause de l'échec de l'institutionnalisation du folklore promue par A. Machado y Álvarez est d'ordre idéologique et par là même plus révélatrice des tensions qui se jouent à ce moment-là en Espagne dans la construction de l'imaginaire national. La conception démocratique et volontariste de l'identité nationale qu'a A. Machado y Álvarez et qui s'exprime dans son projet scientifico-patriotique s'oppose à l'idée de la nation telle que la conçoivent les conservateurs et en particulier leur leader, Antonio Cánovas del Castillo. Celui-ci, dans son Discurso sobre la nación de $1882^{20}$, avait clairement signifié que l'unité de la nation était une question de volonté divine ou de loi naturelle, autrement dit reposait sur des critères intangibles qu'il fallait soustraire au débat et à l'approbation des individus qui la composent. Par ailleurs, pour les tenants du nationalisme conservateur, à l'instar de Marcelino Menéndez Pelayo, la tradition et, à 
travers elle, la connaissance du passé de la nation, doit servir à produire un roman national nostalgique dédié à la glorification des grands hommes, en particulier l'élite monarchique et politique, modèle historiographique opposé à celui que A. Machado y Álvarez entendait mettre en valeur. Or, pendant la phase de développement de El FolkLore Español, les conservateurs et leurs alliés ultramontains ont principalement été au pouvoir et leur inertie de réponse a mis en échec toutes les initiatives promues par A. Machado y Álvarez, dont les velléités fédéralistes et démocratiques allaient à l'encontre de leurs convictions centralisatrices, traditionnalistes et élitistes.

Le projet scientifico-patriotique machadien a également suscité méfiance et rejet chez les partisans des nationalismes périphériques émergents, pour lesquels le folklore doit être mis au service de la documentation de la différence exclusive. Cette divergence explique que les catalanistes ont toujours refusé d'adhérer au réseau machadien de sociétés folkloriques régionales. Par ailleurs, Manuel Murguía, l'impulseur du Primeiro Rexurdimiento, qui avait été collaborateur de A. Machado y Álvarez dans un premier temps et, à ce titre, pressenti pour diriger la société folklorique galicienne quand elle serait créée, a préféré aussi l'entre-soi exclusif pour poser les bases de l'identité galicienne et forger le discours ethno-généalogique du nationalisme galicien, fondé sur le mythe des origines celtiques de la Galice. L'échec de A. Machado y Álvarez à institutionnaliser le folklore à la fin de la première décennie de la Restauration monarchique, et par delà son échec à promouvoir un nationalisme laïc, démocratique et fédéraliste, est révélateur des tensions et des rapports de force qui se jouaient, à ce moment-là, dans la construction de l'imaginaire national en Espagne, tiraillée entre différents styles antagonistes. Cet échec est avant tout dû au contexte politique de la Restauration, même si les contradictions propres au promoteur du projet ont pu avoir une incidence. Cette affirmation est d'autant plus plausible que les outils qu'il proposait (l'institutionnalisation du folklore, les cartes topographiques traditionnelles) ont fonctionné pour asseoir les bases de l'imaginaire national dans des pays voisins, au Portugal et en Irlande, dont les premiers présidents de la république ont été des folkloristes de la génération de A. Machado y Álvarez, en particulier Teófilo Braga. Au Portugal, par exemple, les condisciples du folkloriste espagnol ont pu développer les initiatives (expositions ethnographiques, musée, cartes démographiques traditionnelles) que ce dernier n'avait pu réaliser en Espagne ${ }^{21}$. Quant à Isabelo de los Reyes, l'impulseur du folklore dans l'archipel des Philippines, qui avait été en contact avec collaborateurs de A. Machado y Álvarez ${ }^{22}$, entre autres Alejandro Guichot, et qui, de ce fait, connaissait les conceptions du folkloriste espagnol, s'est prévalu du folklore pour construire l'imaginaire anticolonial de façon à jeter les bases d'un imaginaire national ${ }^{23}$.

\section{NOTES}

1. Selon J. G. Herder, figure tutélaire de la Volkskunde, la littérature populaire en langue vernaculaire est un héritage qui porte la sédimentation de l'histoire dans son expression, caractéristique qui fait d'elle la plus authentique représentante du Volksgeist (l'esprit national). 
Chants, contes, fables et proverbes par lesquels s'exprime la voix du peuple et qui constituent les archives d'une nation sont ainsi l'objet d'un vaste mouvement de collecte dans la première moitié du XIX ${ }^{\mathrm{e}}$ siècle, en particulier en Europe du Nord.

2. Boletin folklórico español, revista quincenal dirigida por Alejandro Guichot y Sierra, Sevilla, Imp. Enrique Rasco, 1885, p. 5.

3. «Análoga ésta, como hemos dicho, a la inglesa, por el objeto principal que persigue, diferénciase, no obstante, de ésta por su carácter y tendencias : la Sociedad española considera los materiales que va a recoger como elementos indispensables para la reconstrucción científica de la historia patria no escrita hasta ahora más que en su parte más externa y política, y eso sólo a retazos y de una manera deficiente y anti-científica », cf. Antonio, Machado y Álvarez (Demófilo ), Obras completas, edición, introducción y notas de Enrique Baltanás, Biblioteca de Autores Sevillanos tome II, n 5, Sevilla, Diputación de Sevilla, Fundación Machado, 2005, p. 1435.

4. «1. Esta Sociedad tiene por objeto recoger, acopiar y publicar todos los conocimientos de nuestro pueblo en los diversos ramos de la ciencia (medicina, higiene, botánica, política, moral, agricultura, etc.) ; los proverbios, cantares, adivinanzas, cuentos, leyendas, fábulas, tradiciones y demás formas poéticas y literarias; los usos, costumbres, ceremonias, espectáculos y fiestas familiares, locales y nacionales; los ritos, creencias, supersticiones, mitos y juegos infantiles en que se conservan más principalmente los vestigios de las civilizaciones pasadas; las locuciones, giros, trabalenguas, frases hechas, motes y apodos, modismos, provincialismos y voces infantiles ; los nombres de sitios, pueblos y lugares, de piedras, animales y plantas, $\mathrm{y}$, en suma, todos los elementos constitutivos del genio, del saber y del idioma patrios, contenidos en la tradición oral $\mathrm{y}$ en los monumentos escritos, como materiales indispensables para el conocimiento y reconstrucción científica do la historia y de la cultura españolas. 2. Esta Sociedad constará de tantos centros cuantas son las regiones que constituyen la nacionalidad española. Estas regiones son: La Castellana (dos Castillas), La Gallega, La Aragonesa, La Asturiana, La Andaluza, La Extremeña, La Leonesa, La Catalana, La Valenciana, La Murciana, La Vasco-Navarra, La Balear, La Canaria, La Cubana, La Puerto-Riqucña y La Filipina. Todas estas regiones, verdaderos miembros del Folk-Lore Español, contraerán la ineludible obligación de dar cuenta de sus trabajos anuales a todos los centros regionales análogos a los que remitirán también un ejemplar por lo menos de todos los periódicos, revistas o libros que publiquen. A excepción de esta obligación y de la aceptación del fin que esta Sociedad se propone, cada centro se constituirá del modo y forma que tenga por conveniente », cf. Antonio, Machdo y Álvarez, « El Folk-Lore Español, sociedad para la recopilación y estudio del saber y de las tradiciones populares. Bases », in Antonio Machado y Álvarez (dir.), El Folk-Lore Andaluz, Sevilla, Francisco Álvarez y Cía , 1882-1883, p. 501-503.

5. Mercedes, Garcia Plata Gomez, «Aux sources du folklore espagnol: le modèle populaire d'Antonio Machado y Álvarez, entre ambition fédératrice et utopie républicaine ", Les Carnets de Bérose, $\mathrm{n}^{\circ}$ 10, Lahic / DPRPS-Direction générale des patrimoines, 2018.

6. Les sociétés folkloriques régionales peuvent, à leur tour, selon les statuts de la fédération, se subdiviser en centres locaux. Toutes les sociétés créées, qu'elles soient régionales ou locales, portent l'appellation «El Folk-Lore » suivi de l'adjectif désignant la nationalité ou l'appartenance géographique, pour l'entité nationale ou les centres régionaux, ou du toponyme pour les locaux (El Folk-Lore de Burguillos), associant ainsi l'étude de la culture populaire à un territoire ou à un terroir. À l'amphibologie du terme originel, qui avait l'inconvénient de désigner à la fois l'objet et le champ disciplinaire, A. Machado y Álvarez, dans sa volonté de le défendre et de le faire accepter, lui a ajouté un troisième sens - et il est le seul à faire cela en Europe -, celui de société folklorique.

7. Mercedes, Garcia Plata Gomez, op. cit., (deuxième partie, chapitres premier et quatrième).

8. La première, El Folk-Lore Andaluz, comprend douze numéros édités entre entre mars 1882 et février 1883 et réunis en un seul volume paru en 1883, a un format assez hétéroclite qui comprend des articles d'analyse, des résultats de collecte, des outils méthodologiques, ainsi 
qu'une partie d'actualité scientifique. La deuxième, El Folk-Lore Español. Biblioteca de la Tradiciones Populares Españolas, qui compte onze volumes publiés entre 1883 et 1886, a davantage pour objet de constituer des archives scientifiques des matériaux collectés.

9. Antonio, Machado y Álvarez (Demófilo), op.cit., tome II, 2005, p. 1518: «Si el idioma y el territorio son dos elementos constitutivos de las nacionalidades ». A. Machado y Álvarez emploie le concept « nationalité » non pas dans son acception juridique, mais ethno-culturelle, propre au XIX ${ }^{\mathrm{e}}$ siècle.

10. Ibid., p. 1514.

11. «Por el Folk-Lore no sólo podemos estudiar el idioma de cada nación, sino su territorio, tal y como es conocido por el pueblo », Ibid., p. 1518.

12. Ibid., p. 1519-1525.

13. Outre différents textes publiés dans les revues folkloriques, la presse, A. Machado y Álvarez a aussi défendu l'intérêt scientifique des cartes topographiques traditionnelles au Congrès de géographie commerciale et mercantile, organisé par J. Costa, en octobre 1883, en présentant la motion suivante: "La necesidad de completar el estudio de nuestra Historia Patria, mediante el conocimiento que el Pueblo, representante del sentido común, de la experiencia continuada y fiel guardador de la tradiciones nacionales, tiene del territorio español, bien se considere este como teatro en que se han verificado todos los hechos que construyen la historia nacional, bien como depositario de todas las energías que, convenientemente explotadas por la ciencia, son la fuente principal de nuestra riqueza pública ", $c f$. Alejandro, Guchot y Sierra, Noticia histórica del folklore [1922], Junta de Andalucía, Consejería de Educación y Ciencia, Colección Educación XXI, Cultura tradicional $n^{\circ} 3,1999$, p. 366.

14. «A esta primera necesidad ocurre, dentro de su esfera de acción, la institución de «El FolkLore »; por él podemos estudiar las tradiciones - «lo que hemos sido» - y las costumbres - «lo que somos aún » : por él estudiamos los sentimientos, ideas, creencias de nuestro pueblo [...] ; por él podemos, reconstituyendo científicamente nuestra historia pasada, conocer y fijar el derrotero de nuestra historia venidera. En esta obra se juntan los amantes de la tradición y los amantes del progreso », cf. Antonio, Machado y Álvarez (Demófilo), op. cit., , p. 1447.

15. Ibid., p. 1448.

16. Mercedes, Garcia Plata-Gomez, op.cit., 2018 (deuxième partie, chapitre quatrième).

17. Benedict, Anderson, L'imaginaire national. Réflexions sur l'origine et l'essor du nationalisme, Paris, La Découverte, 1996, p. 167.

18. Entre autres La América, Revista de España, Boletín de la Institución Libre de Enseñanza, « La Hoja del lunes » de El Imparcial.

19. Antonio Machado y Álvarez avait songé à exploiter les photographies de scènes folkloriques lors d'expositions. Néanmoins, nombre de ses projets et initiatives étant au point mort en raison de l'inertie de l'État en Espagne, le folkloriste espagnol avait proposé cette idée aux Britaniques de la Folk-Lore Society en 1885, cf. Lettre de A. Machado y Álvarez à la Folk-Lore Society : http:// www.berose.fr/?Lettre-a-la-Folk-Lore-Society.

20. Antonio, Cánovas de Castillo, Discurso sobre la nación: inauguración del curso del Ateneo de Madrid, noviembre de 1882, Edición digital basada en la edición de Madrid, Biblioteca Nueva, 1999, p. 107, URL : http://www.cervantesvirtual.com/nd/ark:/59851/bmc6t0j6.

21. João, Leal, Etnografias Portuguesas (1870-1970). Cultura Popular e Identidade Nacional, Lisboa, Publicações Dom Quixote, 2000, p. 33.

22. I sabelo, De los Reyes y Florentino, El Folk-Lore Filipino, Manila, Biblioteca de la «España Oriental », Chofré, 1889.

23. Benedict, Anderson, Les bannières de la révolte. Anarchisme, littérature et imaginaire anticolonial, Paris, La Découverte, 2009. 


\section{RÉSUMÉS}

A. Machado y Álvarez, fils du naturaliste A. Machado y Núñez et petit-neveu de A. Durán, le compilateur du Romancero general, entend institutionnaliser en Espagne le folklore scientifique selon le paradigme de l'école anthropologique britannique. Son héritage intellectuel et familial, ainsi que ses convictions républicaines fédéralistes et son inquiétude pour régénérer la vie intellectuelle espagnole le conduisent à adapter ce modèle exogène à la réalité sociale espagnole et à concevoir le folklore comme un outil scientifique capable d'influer sur la construction de l'imaginaire national. Cependant, son échec à mener à bien cette institutionnalisation, à la fin de la première décennie de la Restauration monarchique, est révélateur des tensions et des rapports de force qui se jouent, à ce moment-là, dans la construction de l'imaginaire national en Espagne, tiraillée entre différents styles antagonistes.

A. Machado y Álvarez, hijo del naturalista A. Machado y Núñez y sobrino nieto de A. Durán, editor de Romancero general, pretende institucionalizar el folklore científico según lo define la escuela antropológica británica. Su herencia intelectual y familiar, así como sus convicciones republicanas federalistas y su inquietud por regenerar la vida intelectual española lo conducen a adaptar este modelo exógeno a la realidad social española y a concebir el folklore como una herramienta capaz de influir en la construcción del imaginario nacional. Sin embargo, el fracaso de su empresa, al final de la primera década de la Restauración monárquica, revela las tensiones y las pugnas que en aquel momento estaban actuando en la construcción del imaginario nacional, la cual se debatía entre diferentes estilos antagónicos.

\section{INDEX}

Mots-clés : folklore, imaginaire national, cartes topographiques traditionnelles, territoire, histoire

Palabras claves : folklore, imaginario nacional, mapas topográficos tradicionales, territorio, historia

\section{AUTEUR}

\section{MERCEDES GÓMEZ-GARCÍA PLATA}

Université Sorbonne Nouvelle, CREC EA 2292 